\title{
Macrophages in inflammation and its resolution
}

\section{Amiram Ariel ${ }^{1 *}$, Isabelle Maridonneau-Parini ${ }^{2,3}$, Patrizia Rovere-Querini ${ }^{4}$, Jerrold S. Levine ${ }^{5}$ and Heiko Müh/ ${ }^{6}$}

1 University of Haifa, Haifa, Israel

${ }^{2}$ CNRS, UMR5089, IPBS (Institut de Pharmacologie et de Biologie Structurale), Toulouse, France

${ }^{3}$ Université de Toulouse, UPS, IPBS, Toulouse, France

${ }^{4}$ San Raffaele Scientific Institute Milano, Milano, Italy

${ }^{5}$ University of Illinois at Chicago, IL, USA

6 University Hospital Goethe University, Frankfurt am Main, Germany

*Correspondence: amiram@research.haifa.ac.il

\section{Edited by:}

Kendall A. Smith, Weill Medical College of Cornell University, USA

Reviewed by:

Kendall A. Smith, Weill Medical College of Cornell University, USA

Macrophages are highly plastic leukocytes that differentiate from monocytes following their entry into extravascular tissues. Macrophages can enter various tissues under inflammatory or non-inflammatory conditions and assume different functions and phenotypes according to the cues they receive from the environment. The notion that inflammation in general and macrophage responses in particular affect physiological phenomena that were previously considered to be not immune-related has enhanced and broadened our understanding of macrophage function during inflammation and its resolution.

This volume brings together 14 manuscripts that cover various aspects of macrophage function during inflammation and its resolution, as well as in several pathologic states for which a significant, long-lasting, macrophage-mediated immune response plays a significant role. Two of the manuscripts present original research on macrophage phagocytosis and its implications. Five provide an overview of macrophage function during inflammation and its resolution, with an emphasis on the modulatory role of particular elements in this response, such as apoptotic leukocytes, specific pathogens, hypoxia, and hormone receptors. The remaining seven manuscripts outline the role of macrophages during inflammation and its resolution in different tissues, including the lung, cardiovascular and adipose tissues, injured skeletal muscle and neuronal tissues, and synovial and oral cavities.

The two original research articles are devoted to the consequences of particle engulfment by macrophages. Labrousse et al. (2011) describe a novel experimental strategy in which they use micro-patterned immune complexes to trigger frustrated phagocytosis and thereby determine spatial parameters in lysosomal movement and fusion. Janko et al. (2011) report on the cumulative binding of CRP and anti-CRP antibodies to the surface of secondary necrotic cells. This binding leads to a pro-inflammatory cytokine response following engulfment by macrophages, implying a potential role for these elements in the etiology of systemic lupus erythematosus.

Of the review articles that discuss the regulation of macrophage differentiation and function by discrete events, two cover the interaction between macrophages and apoptotic leukocytes during the resolution of inflammation. Korns et al. (2011) outline the regulation of apoptotic cell clearance by macrophages (efferocytosis) and the environmental cues that promote the efferocytic capabilities of macrophages. The second manuscript by Ariel and Serhan (2012) reviews the impact of apoptotic cell sensing and disposal by macrophages on the switches in functional phenotypes displayed by these cells. The effect of another environmental factor, hypoxia, on monocyte/macrophage activation, and differentiation through transcriptional and translational modulation is covered by Rahat et al. (2011). Lugo-Villarino et al. (2011) discuss the pathogenesis and co-mortality displayed by two macrophage-inhabiting microbes (HIV and Mycobacterium Tuberculosis) and their influence on macrophage polarization. Patel et al. (2011) review the role of melanocortin receptor expression by macrophages in anti-inflammation and the resolution of inflammation, with attention given to melanocortin receptor agonists as therapeutic agents.

Several review articles discuss the function of macrophages during inflammation and/or its resolution within distinct anatomical sites, taking into account the unique features of these tissue-specific macrophages, in particular the distinct environments in which they reside and their interactions with neighboring cells. Clària et al. (2011) review current knowledge on the contribution of macrophages to the inflammatory state characterizing adipose tissue and the phenotypic changes observed in macrophages during obesity. Kennedy et al. (2011) discuss macrophage polarization occurring within the synovial space of arthritic joints and its modulation by cytokines, transcription factors, and pro-resolving lipid mediators. The article from Bosurgi et al. (2011) describes the multiple actions of macrophages in injured skeletal muscle, where the effects of these cells are a double-edged sword and can either promote healing and repair or lead to fibrosis and fat replacement. Herold et al. (2011) survey the indispensable role of macrophages in the resolution and termination of inflammation in lung infection and injury as well as the molecular pathways involved in these processes. Proper termination of inflammatory events and clearance of apoptotic cells are also critical to the cardiovascular system, as reviewed by Thorp (2012), and defects in macrophage efferocytosis can lead to atherosclerosis and myocardial infarction. While monocyte-derived macrophages 
and resident microglia were previously considered to be detrimental in brain inflammation and injury, recent advances reviewed by Jung and Schwartz (2012) suggest an opposite role for these macrophage-like cells, with a positive impact on brain maintenance and repair. Finally, Hasturk et al. (2012) outline the reciprocal interaction between periodontal disease and chronic inflammatory illnesses and the role that macrophages play in mediating these chronic inflammatory diseases.

\section{REFERENCES}

Ariel, A., and Serhan, C. N. (2012). New lives given by cell death: macrophage differentiation following their encounter with apoptotic leukocytes during the resolution of inflammation. Front. Immun. 3:4. doi: 10.3389/fimmu.2012. 00004

Bosurgi, L., Manfredi, A. A., and Rovere-Querini, P. (2011). Macrophages in injured skeletal muscle: a perpetuum mobile causing and limiting fibrosis, prompting or restricting resolution and regeneration. Front. Immun. 2:62. doi: 10.3389/fimmu.2011.00062

Clària, J., González-Périz, A., LópezVicario, C., Rius, B., and Titos, E. (2011). New insights into the role of macrophages in adipose tissue inflammation and fatty liver disease: modulation by endogenous omega-3 fatty acid-derived lipid mediators. Front. Immun. 2:49. doi: 10.3389/fimmu.2011. 00049

Hasturk, H., Kantarci, A., and Van Dyke, T. E. (2012). Oral inflammatory diseases and systemic inflammation: role of the macrophage. Front. Immun. 3: 118. doi: 10.3389/fimmu.2012.00118

Herold, S., Mayer, K., and Lohmeyer, J. (2011). Acute lung injury: how macrophages orchestrate resolution of inflammation and tissue repair. Front. Immun. 2:65. doi: 10.3389/fimmu.2011.00065

Janko, C., Franz, S., Munoz, L. E., Siebig, S., Winkler, S., Schett, G., et al. (2011). CRP/anti-CRP antibodies assembly on the surfaces of cell remnants switches their phagocytic clearance toward inflammation. Front. Immun. 2:70. doi: 10.3389/fimmu.2011.00070

Jung, S., and Schwartz, M. (2012). Non-identical twins - microglia and monocyte-derived macrophages in acute injury and autoimmune inflammation. Front. Immun. 3:89. doi: 10.3389/fimmu.2012. 00089

Kennedy, A., Fearon, U., Veale, D. J., and Godson, C. (2011). Macrophages in synovial inflammation. Front. Immun. 2:52. doi: 10.3389/fimmu.2011.00052

Korns, D., Frasch, S. C., FernandezBoyanapalli, R., Henson, P. M., and Bratton, D. L. (2011). Modulation of

Altogether, the articles in this volume portray the complexity of the multiple roles played by macrophages and members of their lineage during inflammation and its resolution, and their manipulation by the injured milieu. These topics are currently heavily studied, and advances in the field, facilitated by state-of-the-art genetics and optical technologies, will undoubtedly continue to contribute to our understanding of the immune system's response to foreign insults, trauma, and inflammatory disorders.

macrophage efferocytosis in inflammation. Front. Immun. 2:57. doi: 10.3389/fimmu.2011.00057

Labrousse, A. M., Meunier, E., Record, J., Labernadie, A., Beduer, A., Vieu, C., et al. (2011). Frustrated phagocytosis on micro-patterned immune complexes to characterize lysosome movements in live macrophages. Front. Immun. 2:51. doi: 10.3389/fimmu.2011.00051

Lugo-Villarino, G., Vérollet, C., Maridonneau-Parini I., and Neyrolles, O. (2011). Macrophage polarization: convergence point targeted by Mycobacterium tuberculosis and HIV. Front. Immun. 2:43. doi: 10.3389/fimmu.2011.00043

Patel, H. B., Montero-Melendez, T., Greco, K. V., and Perretti, M. (2011). Melanocortin receptors as novel effectors of macrophage responses in inflammation. Front. Immun. 2:41. doi: 10.3389/fimmu.2011.00041

Rahat, M. A., Bitterman, H., and Lahat, N. (2011). Molecular mechanisms regulating macrophage response to hypoxia. Front. Immun. 2:45. doi: 10.3389/fimmu.2011.00045
Thorp, E. B. (2012). Contrasting inflammation resolution during atherosclerosis and post myocardial infarction at the level of monocyte/macrophage phagocytic clearance. Front. Immun. 3:39. doi: 10.3389/fimmu.2012. 00039

Received: 02 October 2012; accepted: 07 October 2012; published online: 01 November 2012.

Citation: Ariel A, Maridonneau-Parini I, Rovere-Querini P, Levine JS and Mühl $H$ (2012) Macrophages in inflammation and its resolution. Front. Immun. 3:324. doi: 10.3389/fimmu.2012.00324

This article was submitted to Frontiers in Inflammation, a specialty of Frontiers in Immunology.

Copyright (c) 2012 Ariel, MaridonneauParini, Rovere-Querini, Levine and Mühl. This is an open-access article distributed under the terms of the Creative Commons Attribution License, which permits use, distribution and reproduction in other forums, provided the original authors and source are credited and subject to any copyright notices concerning any third-party graphics etc. 